\title{
Resistance Training With Elastic Tubing Improves Muscle Strength, Exercise Capacity, and Post-Exercise Creatine Kinase Clearance in Subjects With COPD
}

\author{
Bruna SA Silva, Dionei Ramos, Carlos A Camillo, Iara B Trevisan, Giovanna A Arévalo, \\ Ana Paula CF Freire, Marceli R Leite, Fabiano F de Lima, Luis A Gobbo, and Ercy MC Ramos
}

BACKGROUND: Among the types of physical exercise, resistance exercises have been gaining significant attention in the COPD population. The aim of this study was to compare effects of conventional resistance training and of training by using elastic tubes on muscle strength, exercise capacity, and creatine kinase clearance in subjects with COPD. METHODS: Twenty-eight subjects with COPD were randomized into the following: resistance training with the elastic tubing group and resistance training with the weight-machine training group (conventional resistance group), performed 3 times a week for 12 weeks. The subjects were submitted to spirometry, functional exercise capacity (the 6-min walk test), muscle strength (dynamometry), and the repetition maximum test. Differences between the initial and final evaluations $(\Delta)$ and the (final - initial evaluations)/initial evaluations $\times 100$ $(\Delta \%)$ of each group were expressed as mean [95\% CI]. RESULTS: Nineteen subjects $\left(\mathrm{FEV}_{1} \%\right.$ predicted, $52 \pm 18$; years, $65 \pm 8$ ) completed the training program. Similar improvements were observed in both modalities on muscle strength (knee extension, $\Delta \% 18$ [6 to 29]; knee flexion, $\Delta \% 35$ [17 to 54]; elbow flexion, $\Delta \% 28$ [ 9 to 48]; shoulder abduction, $\Delta \% 41$ [25 to 58] and shoulder flexion, $\Delta \% 31$ [11 to $51]$ in the weight-machine training group (conventional resistance group); knee extension, $\Delta \% 15$ [8 to 21]; knee flexion, $\Delta \% 28$ [15 to 41]; elbow flexion, $\Delta \% 36$ [22 to 51]; and shoulder abduction, $\Delta \% 43$ [32 to 55] and shoulder flexion, $\Delta \% 43$ [25 to 61] in the elastic tubing group, $P<.05$ for intra-group analysis and $P>.05$ for between groups analysis), 6 -min walk test (baseline $493 \pm 67 \mathrm{~m}$ vs 12 weeks $526 \pm 78$ in the weight-machine training group $(P=.10)$; baseline $493 \pm 71$ vs 12 weeks $524 \pm 68$ in the elastic tubing group $(P<.01), P=.88$ between groups). The elastic tubing group had lower accumulated creatine kinase levels between 24 and $72 \mathrm{~h}((\Delta \%-24[-31$ to 16$]$ than subjects in the weight-machine training group $\Delta \% 3$ [ -21 to 28], $P=.042$ between the groups. CONCLUSIONS: Training with elastic resistance provided similar changes in muscle strength and exercise capacity to conventional resistance group in the subjects with COPD. The elastic tubing group had faster creatine kinase clearance after a training session than the weight-machine training group (conventional resistance group). The ease of its application associated with similar training benefits to conventional training supported its application in clinical routine. Key words: COPD; rehabilitation; exercise training; elastic resistance; muscle strength; creatine kinase. [Respir Care 2019;64(7):835-843. (C) 2019 Daedalus Enterprises]

\section{Introduction}

COPD is primarily a lung disease that features extrapulmonary consequences, such as reduced physical activity,

Ms Silva and Dr Gobbo are affiliated with the Skeletal Muscle Assessment Laboratory, Department of Physical Education, São Paulo State University (UNESP), Presidente Prudente, São Paulo, Brazil. Dr Ramos, increased symptoms in daily life, reduced health-related quality of life, and muscle dysfunction. ${ }^{1-3}$ The dysfunction of the peripheral muscles is seen as an important cause of

Ms Trevisan, Arévalo, Dr Freire, Dr Leite, Mr de Lima, and Dr Ramos
are affiliated with the Department of Physical Therapy, São Paulo State
University (UNESP), Presidente Prudente, São Paulo, Brazil. Dr Camillo University (UNESP), Presidente Prudente, São Paulo, Brazil. Dr Camillo
is affiliated with the University of Londrina, Department of Physiotherapy, 
reduced strength, endurance, and muscle mass, which makes the practice of exercise training an essential component of pulmonary rehabilitation programs. ${ }^{4}$ Exercise training, if sufficiently intense, leads to a significant immune response in healthy individuals mediated by inflammatory cells, hormones, cytokines, and neural factors. ${ }^{5}$ In high-intensity conditions, exercise alters cell membrane permeability and progressively releases creatine kinase (CK) to the circulation, reaching peak levels $\sim 24 \mathrm{~h}$ after the exercise acute session and gradually returning in the and gradually returning in the following hours/ day. ${ }^{6} \mathrm{~A}$ better clearance time of $\mathrm{CK}$ after exercise is also related to better body adaptation to the exercise stimulus. ${ }^{7}$

Among the types of physical exercise, resistance exercises have been gaining significant attention in the COPD population ${ }^{8}$ because the effects go beyond those provided by aerobic exercises ${ }^{9}$ Resistance exercises can be performed in several ways, including conventional resistance training, and training with bands and elastic tubing. ${ }^{10}$ In conventional resistance training, dumbbells, barbells, and weight machine training are generally used. ${ }^{11}$ However, weight machine training is usually expensive, difficult to transport, and requires a considerably large physical space. Elastic tubing is safe, easy to use, portable, and relatively inexpensive. ${ }^{12}$ In addition, these have the ability to increase tension linearly from the start of the contraction until the end of the movement, being less harmful to the joints. ${ }^{11}$ Despite the advantages of elastic resistance compared with conventional resistance training, little is known about the effectiveness of the former in subjects with COPD. ${ }^{13-15}$

Londrina, Paraná, Brazil and University Pitágoras UNOPAR, Departmentt of Rehabilitation Sciences, Londrina, Paraná, Brazil.

This study was performed in São Paulo State University (UNESP), Presidente Prudente, São Paulo, Brazil.

Ms Silva and Ms Arevalo presented the research data at the 25th International Congress European Respiratory Society, 2015, Amsterdam, the Netherlands. Mr de Lima presented the research data at the XVI Congresso Paulista de Pneumologia e Tisiologia, 12 august 2015, Campos do Jordão, Brazil.

The authors have disclosed no conflicts of interest.

Supplementary material related to this paper is available at http:// www.rcjournal.com.

Correspondence: Ercy Mara Cipulo Ramos PT, PhD, Department of Physioterapy, São Paulo State University (UNESP), Rua Roberto Simonsen, No. 305, Presidente Prudente, São Paulo, Brazil 19060-900. E-mail: ercy@bol.com.br.

DOI: $10.4187 /$ respcare. 05975

\section{QUICK LOOK}

\section{Current knowledge}

Peripheral muscle dysfunction is seen as an important cause of reduced muscle strength, impaired creatine kinase function, and functionality in COPD, which makes the practice of resistance training an essential component of pulmonary rehabilitation. However, it is estimated that only $5 \%$ of patients with COPD who could benefit from pulmonary rehabilitation have access to programs.

\section{What this paper contributes to our knowledge}

We demonstrated that elastic tubing was a viable, lowcost alternative tool for resistance training in COPD, with similar improvements in muscle strength and exercise capacity to weight machines. In addition, subjects who exercised with elastic resistance had lower accumulation of muscle stress after a training session.

Our group recently compared the effectiveness of both conventional and elastic resistance training in this population. A potential limitation of the study was the lack of controlled training progression and intensity between the 2 groups. ${ }^{15}$ A study that investigates training effectiveness of elastic resistance (with elastic tubes) compared with conventional resistance training with similar intensity is required. Furthermore, there is no information on whether different approaches of resistance training have a unique impact on CK clearance time. The present study aimed to evaluate the muscle strength, CK clearance, and functional exercise capacity of subjects with COPD submitted to either resistance training with elastic tubing or with weightmachine training. We hypothesized that the resistance training protocol with elastic tubing would improve peripheral muscle strength and functional exercise capacity similarly to resistance training by using weight machines while inducing less muscle stress.

\section{Methods}

The subjects who were clinically stable with moderateto-severe COPD classified according to internationally accepted criteria ${ }^{1}$ underwent a 12 -week program in a specialized rehabilitation center. Knee extension force was a priori defined as the primary end point. Sample-size calculation was done based on equivalence of training. Twenty subjects (10 per group) were needed to achieve a power of $80 \%$, expecting a difference of $45 \mathrm{~N}$ between groups with a SD of $36 \mathrm{~N}$ and taking into account a typical dropout rate of $20 \%$. Patients were excluded if they were 


\section{Elastic vs Conventional Strength Training in COPD}

active smokers or presented with unstable cardiac disease or musculoskeletal disorders that would prevent implementing the experimental protocol. Patients were also excluded if they had an exacerbation or complications that hindered the continuity of the treatment.

All the individuals were informed beforehand of the objectives and procedures of the study and provided informed written consent to participate in the study. All procedures were approved by the local ethics committee, São Paulo State University (UNESP), Presidente Prudente, São Paulo, Brazil (CAAE 12492113.5.0000.5402) and followed Resolution 466/12 of the Brazilian National Health Council. Recruitment and follow-up of individuals was conducted between 2014 and 2015 in a rehabilitation center in Presidente Prudente, São Paulo, Brazil. This radomized trial is registered with the Brazilian Registry of Clinical Trials (Universal Trial RBR-7kcr2p).

\section{Study Design}

Individuals were randomized by strata as follows: first, the subjects were classified into quartiles according to individual relative strength of the lower limbs (maximum voluntary isometric contraction of the knee extension). ${ }^{16}$ Next, individuals in each quartile were randomized by numbered brown envelopes by an individual who was not related to the study, into one of the two groups a priori defined as follows: (1) either to perform resistance training with elastic tubing (ET group) or (2) to perform conventional resistance training (CR group). Although the therapists ( BS, FL and GA) and subjects were not blinded to the interventions, data analysis was performed by an individual who was not involved with the data collection. The subjects were submitted to an initial evaluation, which included measurements of expired carbon monoxide, ${ }^{17}$ lung function (spirometry), ${ }^{1,18-20}$ functional exercise capacity (the 6-min walk test [6MWT]), muscle strength of the upper and lower limbs (dynamometry), and the numberof-repetitions test by using conventional weight-machine training in the $\mathrm{CR}$ group and elastic tubing in the ET group. On completion of the 12-week training period, the same evaluation was repeated to verify the training effects. Finally, muscle stress (via serum levels of CK) ${ }^{21}$ was assessed at baseline and in the final week.

\section{Pulmonary Function}

Spirometry was performed by using a digital spirometer (Medical International Research (MIR)-Spirobank version 3.6, Roma, Italy) according to guidelines for pulmonary function tests. ${ }^{20}$ The interpretation was performed by using recommendations of the American Thoracic Society and the European Respiratory Society, ${ }^{18}$ and the results were compared with normative data of a Brazilian popu- lation. ${ }^{19}$ The spirometric criterion for air-flow limitation was a postbronchodilator fixed ratio $\mathrm{FEV}_{1} / \mathrm{FVC}$ of $<0.70$. Classification of COPD severity followed the Global Initiative for Chronic Obstructive Lung Disease. ${ }^{1}$

\section{Analysis of Muscle Stress}

CK activity was assessed at the following moments: week 0 (before the training session) and weeks $12(24,48$, and $72 \mathrm{~h}$ after the last training session) from a blood sample collected from the tip of the index finger of the individual. A $30-\mu \mathrm{L}$ sample of fresh blood was collected in a heparinized capillary tube and then immediately pipetted onto the CK test strip directly from the capillary tube. The analysis was performed by using a colorimetric assay procedure with Reflotron (Boehringer, Mannheim, Germany); the unit of measurement was U/L. ${ }^{21}$

\section{Functional Exercise Capacity}

The functional exercise capacity was evaluated by using the 6MWT according to the guidelines of the European Respiratory Society/American Thoracic Society. ${ }^{22}$

\section{Muscle Strength and Load Increment Over the Sessions}

The measurement of strength was performed on the dominant side, at baseline, and after 6 weeks and 12 weeks of training by using a digital dynamometer (Force Gauge, model FG-5100, Shanghai, China), with results expressed in newtons $(\mathrm{N})$. The following muscle groups were evaluated: knee flexors and extensors, shoulder flexors and abductors, and elbow flexors. ${ }^{10}$

The criterion to increase work load was based on the number-of-repetitions test (NR) performed at the beginning of each session. Participants performed the numberof-repetitions test to verify the maximum number of repetitions that they could perform with a given load. The load would be maintained for that session when the maximum number of repetitions performed was $15 \pm 2$; otherwise, it would be adjusted to achieve the expected number of repetitions. ${ }^{16}$ The increment in the ET group was done by changing the diameter of the tubes and/or by adding extra tubes. Increases in the work load for subjects in the CR group followed the same criterion of the ET group with changes in the weights of the machine. The measurement of the load in the ET group was performed according to a previous study. ${ }^{23}$

\section{Training Protocol Programs Offered to the ET and CR Groups}

Both groups followed the training programs for 12 weeks (3 times per week), which totaled 36 training sessions of 
$\sim 60$-min durations. At the beginning and at the end of each session, the vital signs were verified and stretching of the trained muscle groups was carried out. Before the start of the training, the participants were familiarized with the exercises, equipment, and elastic tubing. The movements were performed in the following order: shoulder abduction, elbow flexion, shoulder flexion, knee extension, and knee flexion. Thus, the muscles trained were deltoid, coracobrachial, pectoralis major, supraspinatus, brachialis, biceps brachialis, brachioradialis, biceps femoris, semimembranosus, semitendinosus, and quadriceps femoris. Exercises were conducted by using specific time frames and a progressive design starting with $2 \times 15 \mathrm{NR}$ (weeks 1-3), $3 \times 15 \mathrm{NR}$ (weeks 4-6), $3 \times 10 \mathrm{NR}$ (weeks $7-9$ ), and $4 \times 6 \mathrm{NR}$ (weeks 10-12) with a 2-min interval between sets. The training program was developed in our study center, and more details of the adopted training protocols can be found in the literature. ${ }^{24}$

\section{Resistance Training With Elastic Tubing}

Elastic tubing was used for the training in the ET group. Different tubes with progressive resistance were used. The higher the reference number, the higher the diameter (resistance) of the tube (nos. 200, 201, 202, 203, and 204; Lemgruber, Rio de Janeiro, Brazil). Exercises were performed by using a specific chair that had an elastic tubing support fitting for each trained muscle group. ${ }^{12,24}$ In addition, metal rings and beams (plastic cable ties) were used for fixating the elastic tubing to the rings. Based on a pilot study (unpublished data), we defined the initial selection of a tube diameter for each movement by using subjects' dynamometry. The initial lengths for the tubing were determined according to each individual's distance from the upper or lower limb to the hook (fixed point) on the chair. Thus, the length of the tubing used for each subject was unique and remained the same in all sessions. Trained muscle groups were the same as those evaluated during dynamometry (see the supplementary material at http:// www.rcjournal.com).

\section{Conventional Resistance Training}

The duration of conventional resistance training and the progression were similar to the method offered to the ET group. A weight machine (Ipiranga-model Executive (adapted), Rio de Janeiro, Brazil) was used for the conventional resistance training. Simple pulley equipment was used for the upper-limb training, whereas open-chain single-legged flexion-extension exercises were performed for the lower limbs. ${ }^{12}$

\section{Statistical Analysis}

A statistical package (SPSS 22, SPSS, Chicago, Illinois) was used for data analysis. Normality of the data was assessed by using the Shapiro-Wilk test, and results were described as mean \pm SD or as median (25-75\% interquartile range) according to the data distribution. Differences between the initial and final evaluations $(\Delta)$ and the (final - initial evaluations)/initial evaluations $\times 100(\Delta \%)$ of each group were expressed as mean $(95 \% \mathrm{CI})$. Analysis of variance for repeated measures model in a 2-factor scheme was performed for each group to compare training effects on the different time points (week 0 , weeks 6 , and weeks 12). Data used for the repeated measurements were checked for violation of sphericity by using the Mauchly test. The Greenhouse-Geisser correction was used when the sphericity was violated. For intragroup analysis of the moments (baseline, 6 weeks, and 12 weeks), the Bonferroni posttest was used for parametric distribution or the Dunnet posttest for nonparametric distribution. The comparison of the initial moments and percentage variations in gains between the groups at baseline and 12 weeks moments was performed by using the Student $t$ test for parametric distributions or the Mann-Whitney test for nonparametric distributions. Categorical variables were analyzed by using the chi-square test. The level of significance was set at $P<.05$.

\section{Results}

A complete overview of the study can be seen in Figure 1. Twenty-eight subjects with COPD were included and allocated into one of the two groups. Five subjects discontinued the training after commencing the program, and one was excluded from final analysis because the subject returned to smoking. Randomization according to individual relative muscle strength assured similarity in baseline strength levels between the training groups (ET group, $242 \pm 46 \mathrm{~N}$; CR group, $247 \pm 62 \mathrm{~N} ; P=.40$ ). Subject characteristics are presented in Table 1. No significant differences were found between the groups.

The progression of the training load in the 12 weeks of training in the 2 groups is presented in Figure 2. Although the progression of training load in both groups was based on the same criteria (ie, number-of-repetitions test), the subjects in the ET group managed greater work load progression in elbow flexion and in knee flexion-extension exercises than the subjects did in the CR group from weeks 6-7 onward (elbow flexion $[P=.035]$, knee flexion $[P=.045]$, and knee extention $[P=.01])$. Training effects on muscle function can be seen in Table 2. No statistical differences were observed between the groups at baseline. A significant increase in strength can be observed in all movements analyzed in both groups. In ad- 


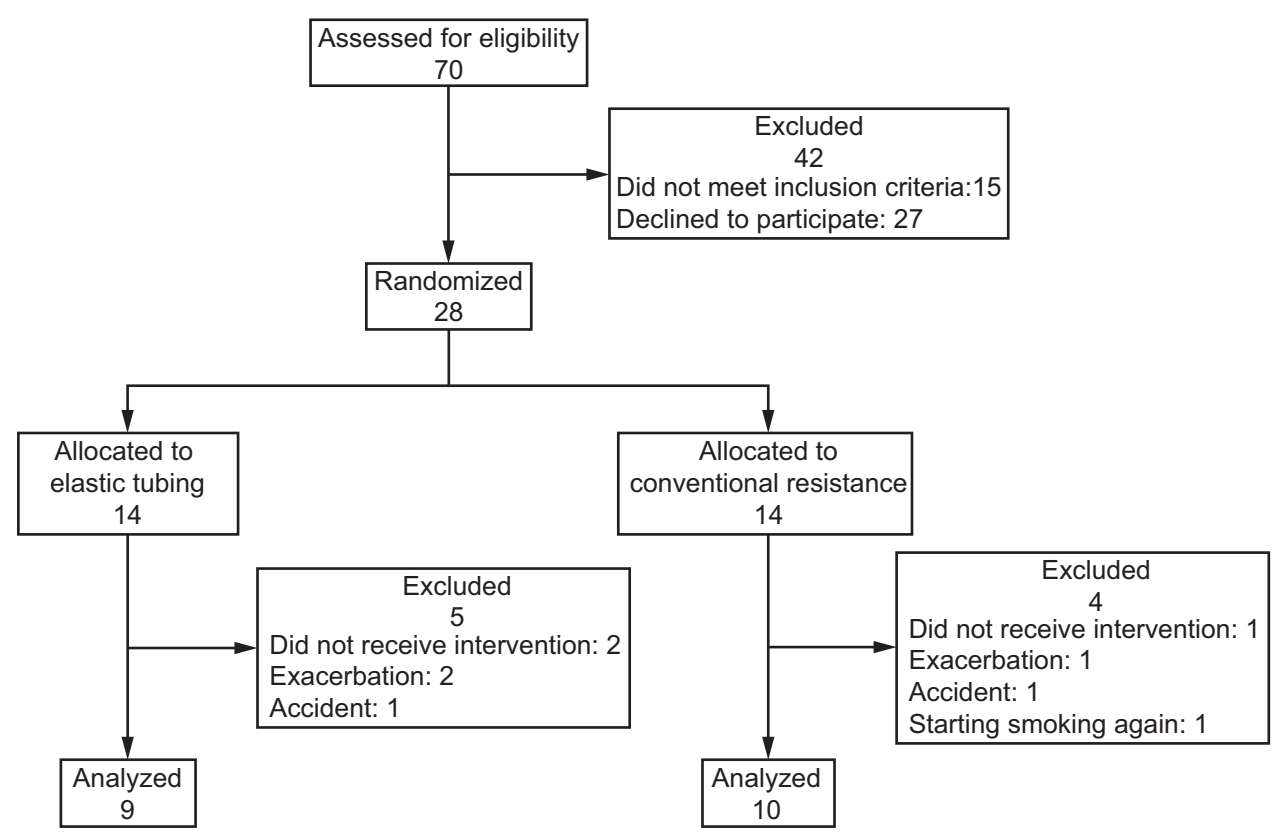

Fig. 1. Flow chart.

Table 1. Baseline Characteristics of the Subjects by Group. Data Expressed in Mean SD or Median (IQR)

\begin{tabular}{|c|c|c|c|}
\hline Characteristic & $\begin{array}{c}\text { CR Group } \\
(n=14)\end{array}$ & $\begin{array}{l}\text { ET Group } \\
(n=14)\end{array}$ & $P$ \\
\hline Age, mean $\pm \mathrm{SD}$ y & $65 \pm 8$ & $63 \pm 9$ & .40 \\
\hline Males, $n(\%)$ & $10(71)$ & $9(64)$ & .69 \\
\hline BMI, median (IQR) $\mathrm{kg} / \mathrm{m}^{2}$ & $28(23-32)$ & $27(22-33)$ & .75 \\
\hline FVC\%, median (IQR) & $78(73-85)$ & $66(50-86)$ & .14 \\
\hline $\mathrm{FEV}_{1} \%$, median (IQR) & $59(42-71)$ & $37(35-64)$ & .12 \\
\hline GOLD II/III/IV & 06/07/01 & 04/08/02 & .67 \\
\hline $\mathrm{CO}$, median (IQR) ppm & $0(0-3)$ & $2(0-3)$ & .28 \\
\hline $\mathrm{HbCO}$, median (IQR) \% $\mathrm{Hb}$ & $0(0-3)$ & $0.32(0-0.52)$ & .28 \\
\hline $\begin{array}{l}\mathrm{CR}=\text { conventional resistance } \\
\mathrm{ET}=\text { elastic tubing } \\
\mathrm{BMI}=\text { Body mass index } \\
\mathrm{IQR}=25-75 \% \text { interquartile range } \\
\mathrm{GOLD}=\text { Global Initiative for Chroni } \\
\mathrm{CO}=\text { Carbon monoxide } \\
\mathrm{ppm}=\text { parts per million } \\
\mathrm{HbCO}=\text { carboxyhemoglobin } \\
\mathrm{Hb}=\text { hemoglobin }\end{array}$ & structive Lung D & & \\
\hline
\end{tabular}

dition, no differences were observed on the magnitude of 6MWT changes in the evaluated groups. However, when analyzed individually, the ET group had slightly larger improvements than the CR group in the 6MWT from baseline.

The behavior of the serum levels of CK from baseline to the measurements at $72 \mathrm{~h}$ is depicted in Figure 3. There was an effect between moments (baseline and 24, 48, and $72 \mathrm{~h})(P=.02$, effect size $=0.18)$. Both groups had similar increases in CK levels from baseline to $24 \mathrm{~h}$
( $\Delta \% 33[-5$ to 71$]$ for the ET group and $\Delta \% 32$ [14-50] for the CR group, $P=.96$ between the ET and CR group). Differences between CK measured at baseline and $72 \mathrm{~h}$ were not significant between the groups $(P=.15$, ET group, $\Delta \% 2$ [ -31 to 37]; CR group, $\Delta \% 39$ [ -3 to 81]). However, the subjects in the ET group had a faster reduction of $\mathrm{CK}$ levels between 24 and $72 \mathrm{~h}(\Delta \%-24$ [ -31 to 16$]$ for the ET group and $\Delta \% 3[-21$ to 28$]$ for the $\mathrm{CR}$ group; $P=.042$ between the groups).

\section{Discussion}

The main findings of the present study demonstrated that both training regimens improved peripheral muscle strength and functional exercise capacity similarly. In addition, the subjects in the ET group showed a faster reduction of the muscle stress after a training session at the end of the 12 weeks of training. Elastic resistance training is commonly used by athletes due to its highly adaptable characteristics with the additional advantage of inducing reduced levels of muscle stress (preventing overtraining). As a consequence, it renders better individual performance and training effects. ${ }^{25}$ It is also relatively inexpensive and easy to use, which increases its dissemination and its use in clinical practice. Despite the benefits, it is not widely used in pulmonary rehabilitation programs.

Previous work describes modest benefits of elastic resistance training using elastic bands (Theraband, Akron, Ohio, EUA) on muscle strength in subjects with COPD after a partially supervised domiciliary training program of 12 weeks. ${ }^{14}$ Nyberg et al ${ }^{13}$ showed benefits of elastic resis- 

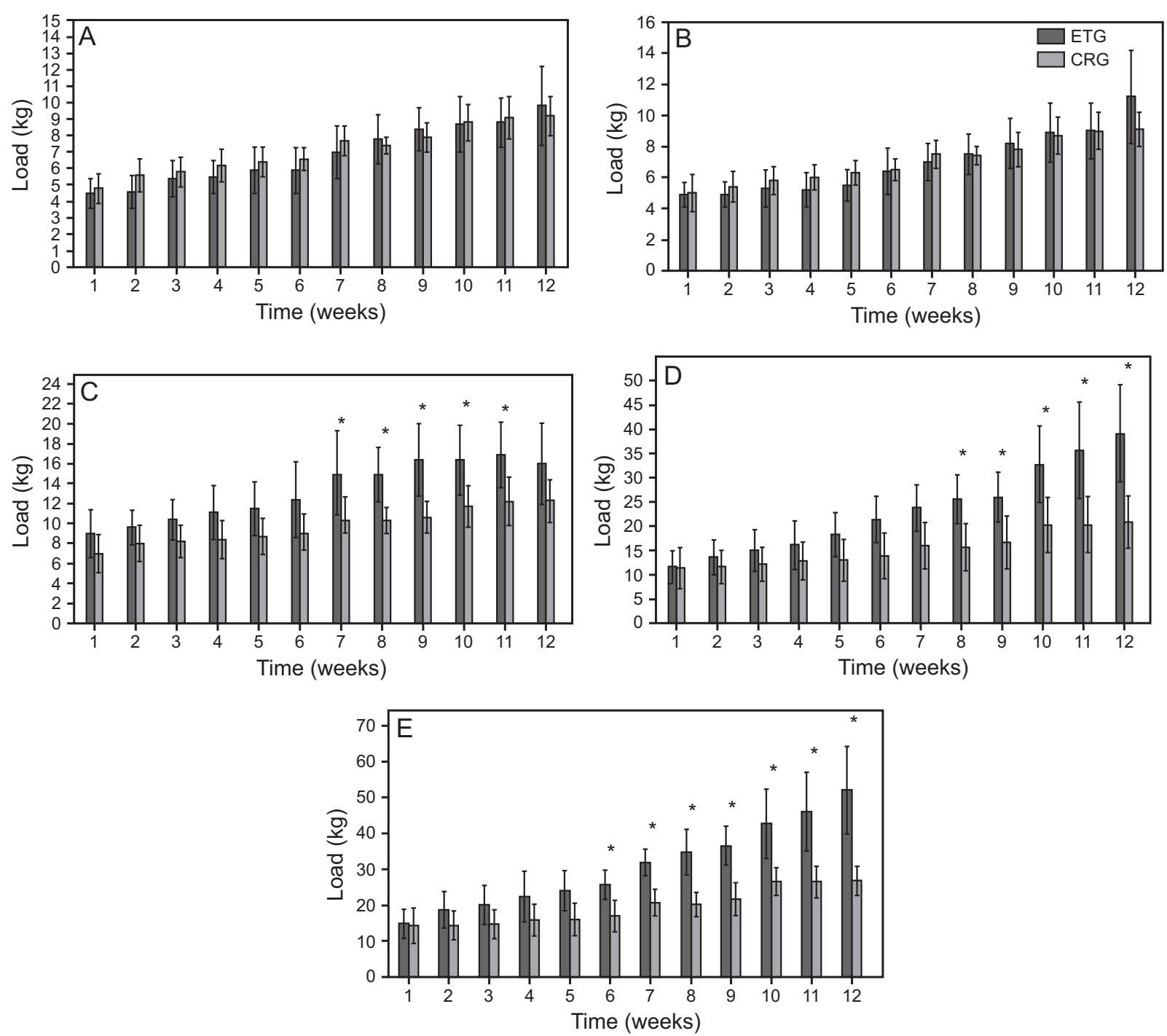

Fig. 2. Training progression during the 12 weeks of training in both groups. Bars show mean \pm SD. A: Shoulder abduction; B: shoulder flexion; C: elbow flexion; D: knee flexion; E: knee extension. ET: elastic tubing resistance training group; CR: conventional weight machine resistance training group. *Significant difference between groups.

tance on functional exercise capacity and muscle function in subjects with moderate-to-severe COPD when compared with a control group. In a recent study, our group demonstrated benefits of an 8-week training protocol when using elastic tubing compared with using a weight machine in subjects with COPD. ${ }^{15}$ A point of criticism of the latter was the limited comparability of the delivered work load during training protocols. In the present study, we confirmed these findings by using a 12 -week program with comparable training progression and expanded the results by also describing benefits on serum levels of muscle stress in subjects with COPD. ${ }^{15}$

Although the progression of the training load in both groups was based on the same criteria, subjects in the ET group progressed more than subjects in the CR group from weeks 6-7 onward, especially in the lower limbs. This result was not expected, but we speculated that the higher activation of muscle units promoted by elastic resistance compared with weight-machine resistance ${ }^{11}$ may have contributed. The possible higher recruitment of muscle fibers in the ET group may have influenced the subjects' effort perception in relation to the training loads, in which higher loads could be supported even with effort perception similar to the CR group. This higher recruitment of muscle fibers can be related to the property of elastic resistance to increase tension linearly from the start of the contraction until the end of the movement. ${ }^{11}$ Muscle strength was significantly improved in both groups after 12 weeks of training with effects mostly observed in the first 6 weeks of training. This was in line with a recent meta-analysis that demonstrated a minimum of 4 weeks of training as necessary to promote clinically important gains in functional exercise capacity and health-related quality of life. ${ }^{26}$ 


\section{Elastic vs Conventional Strength Training in COPD}

Table 2. Muscle Strength and Functional Exercise Capacity of the Groups Subjected to the Elastic Tubing and Conventional Training at the Evaluated Moments

\begin{tabular}{|c|c|c|c|c|c|c|c|}
\hline Factor & Baseline & 6 Weeks & 12 Weeks & $P^{*}$ & Effect Size & $\Delta \% 0-12$ Weeks & $P \dagger$ \\
\hline \multicolumn{8}{|l|}{ Muscle strength } \\
\hline CR group & $248 \pm 69 \ddagger$ & $282 \pm 78$ & $288 \pm 75 \S$ & & 0.56 & 18 (6 to 29$)$ & \\
\hline Knee extension, $\mathrm{N}$ & & & & $<.001$ & & & .91 \\
\hline ET group & $243 \pm 56 \ddagger$ & $261 \pm 56 \|$ & $277 \pm 56 \S$ & & 0.61 & 15 (8 to 21$)$ & \\
\hline CR group & $137(113-165) \ddagger$ & $176(151-212)$ & $180(153-216) \S$ & & 1.11 & 35 (17 to 54$)$ & \\
\hline Knee flexion, $\mathrm{N}$ & & & & $<.001$ & & & .47 \\
\hline ET group & $127(111-157) \ddagger$ & $174(150-190)$ & $173(146-193) \S$ & & 1.16 & $28(15$ to 41$)$ & \\
\hline CR group & $53(40-67) \ddagger$ & $64(52-79) \|$ & $70(58-87) \S$ & & 1.00 & $41(25$ to 58$)$ & \\
\hline Shoulder abduction, $\mathrm{N}$ & & & & $<.001$ & & & .82 \\
\hline ET group & $49(44-69) \ddagger$ & $70(64-84)$ & $73(67-92) \S$ & & 1.44 & 43 (32 to 55$)$ & \\
\hline CR group & $63 \pm 20 \ddagger$ & $74 \pm 18 \|$ & $78 \pm 18 \S$ & & 0.79 & $31(11$ to 51$)$ & \\
\hline Shoulder flexion, $\mathrm{N}$ & & & & $<.001$ & & & .13 \\
\hline ET group & $65 \pm 19 \ddagger$ & $79 \pm 21 \|$ & $90 \pm 17 \S$ & & 1.39 & $43(25$ to 61$)$ & \\
\hline CR group & $102 \pm 33 \ddagger$ & $114 \pm 35 \|$ & $125 \pm 32 \S$ & & 0.71 & 28 (9 to 48$)$ & \\
\hline Elbow flexion, $\mathrm{N}$ & & & & $<.001$ & & & .32 \\
\hline ET group & $94 \pm 35 \ddagger$ & $113 \pm 33 \|$ & $123 \pm 37 \S$ & & 0.81 & $36(22$ to 51$)$ & \\
\hline \multicolumn{8}{|l|}{ Functional exercise capacity } \\
\hline CR group & $493 \pm 67$ & ND & $526 \pm 78$ & .10 & 0.46 & $7(-3$ to 18$)$ & \\
\hline 6MWD, m & & & & & & & .88 \\
\hline ET group & $493 \pm 71$ & ND & $524 \pm 68$ & $<.001$ & 0.45 & 7 (4 to 9$)$ & \\
\hline \multicolumn{8}{|c|}{ 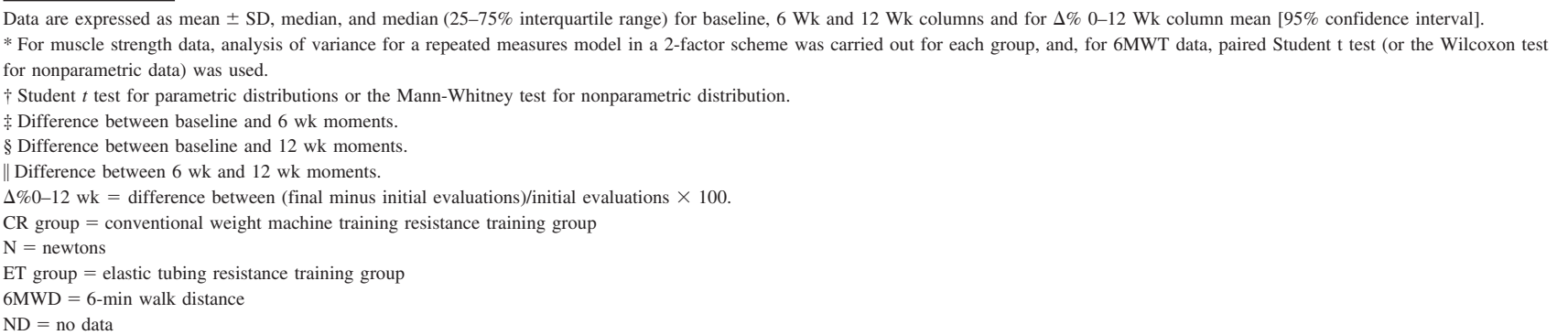 } \\
\hline
\end{tabular}

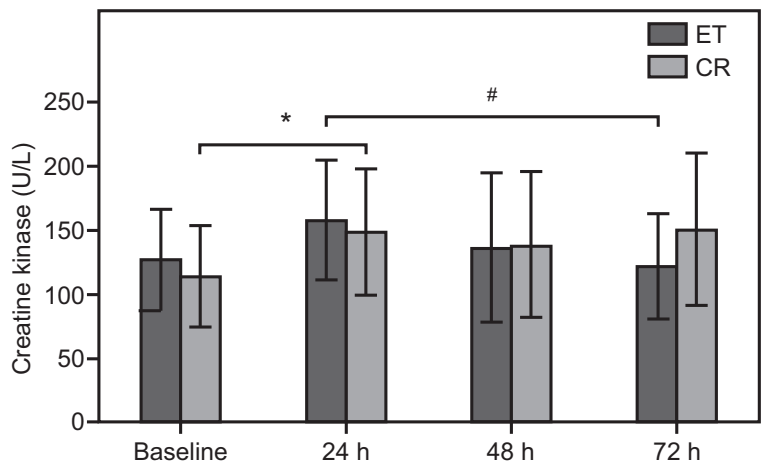

Fig. 3. Creatine kinase behavior in the evaluated training. ET = elastic tubing resistance training group; $\mathrm{CR}=$ conventional weightmachine resistance training group; *Significant difference between the baseline time and $24 \mathrm{~h}$ in CR group; \#Significant difference between times 24 and $72 \mathrm{~h}$ in ET group.

Despite including a sample-size calculation, there was no statistical improvement in the 6MWT after conventional treatment, which was not expected. Commonly, re- sistance training induces positive changes in functional exercise capacity. ${ }^{22,27}$ However, no differences were observed on the magnitude of 6MWT changes between the evaluated groups. Likewise, the changes in both groups exceeded, on average, values above those considered as minimally important differences in the test $(30 \mathrm{~m})^{28}$ and the proportion of subjects in both groups that reached the minimally important differences of the test was similar $(P=.59)$. This information supported the equivalence of both modalities on changes in the 6MWT. We could not exclude the possibility that the difference in training loads between the groups could have a positive effect on the intragroup improvement of the ET group compared with the CR group, but the equivalence of minimally important differences mentioned above added to the fact that there was no difference in the gains $(\Delta \%)$ allowed us to speculate that the same results would be found in the CR group in a study that included a larger sample size (powered for this outcome). 


\section{Elastic va Conventional Strength Training in COPD}

$\mathrm{CK}$ is an enzyme that plays an important role in energy metabolism within muscles and is predominantly present in the muscle tissue. ${ }^{29}$ When the intensity of exercise exceeds the metabolic capacity, there is a significant change in cell membrane permeability and CK is released from within cells to the circulation, with consequent elevation of its serum levels. ${ }^{29}$ An increase of the serum levels of CK observed in both groups $24 \mathrm{~h}$ after the exercise session confirmed that, even at the final stages of the training (week 12), the delivered intensity was high enough to induce overload. This was in line with previous findings in COPD. ${ }^{30}$

Ideally, serum CK levels should quickly return to baseline and thus avoid accumulation in subsequent sessions. ${ }^{7}$ Our results showed that, in comparison with peak values of CK (measured $24 \mathrm{~h}$ after a training session), CK clearance after $72 \mathrm{~h}$ was faster in the ET group. Of note, this phenomenon occurred despite the higher work loads at the last weeks of training in the ET groups. Elastic resistance tends to induce higher muscle activation in comparison with conventional muscle resistance, ${ }^{11}$ and this greater activation decreases the risk of muscle damage because more fibers are recruited simultaneously. Therefore, it can be speculated that muscle damage is reduced, which justifies a faster CK clearance in the ET group. Because the investigation of causes related to increased clearance after the training modalities are out of the scope of the present investigation, this hypothesis remains to be confirmed in a future investigation.

Finally, it is important to note that this study was powered to find differences in muscle strength and was innovative in including the elastic tubing load measurement, which is not commonly performed in elastic resistance training and that facilitates the comparison of training tools. As limitations, despite the sample size calculation for the primary outcome (muscle strength), it was not possible to confirm whether the sample included in this study had enough power for the effects on any of the secondary outcomes. Therefore, the observed effects on functional exercise capacity and muscle stress need to be confirmed in a large study powered for these specific outcomes. In addition, we did not perform intention-to-treat analysis; nevertheless, the power of the study was not impaired because the subjects who completed this study followed our sample-size calculation.

\section{Conclusions}

Based on these results, we concluded that training with elastic resistance provided similar changes in muscle strength and exercise capacity to a conventional resistance training program in subjects with COPD. The subjects in the ET group had faster CK clearance after a training session than did the CR group. The ease of its application associated with similar training benefits to conventional training supports its application in clinical routine.

\section{REFERENCES}

1. Global Initiative for Chronic Obstructive Lung Disease - GOLD. Global Strategy for the Diagnosis, Management, and Prevention of COPD, global Initiative for Chronic Obstructive Lung Disease (GOLD) 2017. Available at www.goldcopd.org. Accessed November 3, 2017.

2. Mathers CD, Loncar D. Projections of global mortality and burden of disease from 2002 to 2030. PLoS Med 2006;3(11):e442.

3. Spruit MA, Singh SJ, Garvey C, ZuWallack R, Nici L, Rochester C, et al.; ATS/ERS Task Force on Pulmonary Rehabilitation. An official American Thoracic Society/European Respiratory Society statement: key concepts and advances in pulmonary rehabilitation. Am J Respir Crit Care Med 2013;188(8):e13-64.

4. Ries AL, Bauldoff GS, Carlin BW, Casaburi R, Emery CF, Mahler DA, et al. Pulmonary Rehabilitation: Joint ACCP/AACVPR Evidence-Based Clinical Practice Guidelines. Chest 2007;131(5):4S-42S.

5. Pedersen BK, Hoffman-Goetz L. Exercise and the immune system: regulation, integration, and adaptation. Physiol Rev 2000;80(3):10551081.

6. Schols AM, Soeters PB, Dingemans AM, Mostert R, Frantzen PJ, Wouters EF. Prevalence and characteristics of nutritional depletion in patients with stable COPD eligible for pulmonary rehabilitation. Am Rev Respir Dis 1993;147(5):1151-1156.

7. Ehlers GG, Ball TE, Liston L. Creatine kinase levels are elevated during 2-a-day practices in collegiate football players. J Athl Train 2002;37(2):151-156.

8. Alison JA, McKeough ZJ. Pulmonary rehabilitation for COPD: are programs with minimal exercise equipment effective? J Thorac Dis 2014;6(11):1606-1614.

9. Strasser B, Siebert U, Schobersberger W. Effects of resistance training on respiratory function in patients with chronic obstructive pulmonary disease: a systematic review and meta-analysis. Sleep Breath 2013;17(1):217-226

10. Ricci-Vitor AL, Bonfim R, Fosco LC, Bertolini GN, Ramos EM, Ramos D, et al. Influence of the resistance training on heart rate variability, functional capacity and muscle strength in the chronic obstructive pulmonary disease. Eur J Phys Rehabil Med 2013;49(6): 793-801.

11. Melchiorri G, Rainoldi A. Muscle fatigue induced by two different resistances: Elastic tubing versus weight machines. J Electromyogr Kinesiol 2011;21(6):954-959.

12. Lima FF, Camillo CA, Gobbo LA, Trevisan IB, Nascimento WBBM, Silva BSA, et al. Resistance training using low cost elastic tubing is equally effective to conventional weight machines in middle-aged to older healthy adults: a quasi-randomized controlled clinical trial. J Sports Sci Med 2018;17(1):153-160.

13. Nyberg A, Lindström B, Rickenlund A, Wadell K. Low-load/highrepetition elastic band resistance training in patients with COPD: a randomized, controlled, multicenter trial. Clin Respir J 2015;9(3): 278-288.

14. O'Shea SD, Taylor NF, Paratz JD. A predominantly home-based progressive resistance exercise program increases knee extensor strength in the short-term in people with chronic obstructive pulmonary disease: a randomised controlled trial. Aust J Physiother 2007; 53(4):229-237.

15. Ramos EM, de Toledo-Arruda AC, Fosco LC, Bonfim R, Bertolini GN, Guarnier FA, et al. The effects of elastic tubing-based resistance training compared with conventional resistance training in patients with moderate chronic obstructive pulmonary disease: a randomized clinical trial. Clin Rehabil 2014;28(11):1096-1106. 


\section{Elastic vs Conventional Strength Training in COPD}

16. Persch LN, Ugrinowitsch C, Pereira G, Rodacki AL. Strength training improves fall-related gait kinematics in the elderly: a randomized controlled trial. Clin Biomech (Bristol, Avon) 2009;24(10):819-825.

17. Santos UP, Gannam S, Abe JM, Esteves P, Freitas FM, Wakassa TB, et al. Use of breath carbon monoxide as an indicator of smoking status. Jornal de Pneumologia 2001;27(5):231-236.

18. Miller MR, Hankinson J, Brusasco V, Burgos F, Casaburi R, Coates A, et al.; ATS/ERS Task Force. Standardisation of spirometry. Eur Respir J 2005;26(2):319-338.

19. Neder JA, Andreoni S, Castelo-Filho A, Nery LE. Reference values for lung function tests. I. Static volumes. Braz J Med Biol Res 1999;32(6):703-717.

20. Carlos ACP, Jansen JM, Barreto SSM, Marinho J, Sulmonett N, Dias RM, et al. Diretrizes para Testes de Função Pulmonar [Guideline published in Brazilian Journal of Pulmonology]. Jornal Brasileiro de Pneumologia 2002;3(28):2-237.

21. Twist $\mathrm{C}$, Eston R. The effects of exercise-induced muscle damage on maximal intensity intermittent exercise performance. Eur J Appl Physiol 2005;94(5-6):652-658.

22. Holland AE, Spruit MA, Troosters T, Puhan MA, Pepin V, Saey D, et al. An official European Respiratory Society/American Thoracic Society technical standard: field walking tests in chronic respiratory disease. Eur Respir J 2014;44(6):1428-1446.

23. Lima FF, Camillo CA, Reis EAPD, Job AE, Silva BSA, Topalovic $\mathrm{M}$, et al. Mechanical properties, safety and resistance values of
Lemgruber® elastic tubing. Braz J Phys Ther 2018. pii: S14133555(17)30437-9.

24. Silva BSA, Gobbo LA, Freire APCF, Trevisan IB, Silva IG, Ramos EMC. Effects of a resistance training with elastic tubing in strength, quality of life and dyspnea in patients with chronic obstructive pulmonary disease. J Phys Educ [online] 2016;27:e2722.

25. American College of Sports Medicine. American College of Sports Medicine position stand. Progression models in resistance training for healthy adults. Med Sci Sports Exerc 2009;41(3):687-708.

26. COPD Working Group. Pulmonary rehabilitation for patients with chronic pulmonary disease (COPD): an evidence-based analysis. Ont Health Technol Assess Ser 2012;12(6):1-75.

27. Spruit MA, Gosselink R, Troosters T, De Paepe K, Decramer M. Resistance versus endurance training in patients with COPD and peripheral muscle weakness. Eur Respir J 2002;19(6):1072-1078.

28. Probst VS, Troosters T, Pitta F, Decramer M, Gosselink R. Cardiopulmonary stress during exercise training in patients with COPD. Eur Respir J 2006;27(6):1110-1118.

29. Brancaccio P, Maffulli N, Buonauro R, Limongelli FM. Serum enzyme monitoring in sports medicine. Clin Sports Med 2008;27(1): 1-18, vii.

30. Guzun R, Aquilaniu B, Wuyam B, Mezin P, Koechlin-Ramonatxo $\mathrm{C}$, Auffray $\mathrm{C}$, et al. Effects of training at mild exercise intensities on quadriceps muscle energy metabolism in patients with chronic obstructive pulmonary disease. Acta Physiol (Oxf) 2012;205(2): 236-246. 